\title{
P2P File Transfer System Based on NFC
}

\author{
Jia-Xin Yue 1, a, Zhong Wang 2,b \\ ${ }^{1}$ Dept. of Drama Film and Television Art Design, Sichuan Film and Television University \\ Chengdu, China \\ ${ }^{2}$ College of Electronic Information, Sichuan University ,Chengdu, China \\ ayuejiaxin2017@163.com, b w_linlin518@163.com
}

\begin{abstract}
Keywords: Simple NDEF protocol; P2P communication; Android Beam; file transfer; Android application development.
\end{abstract}

Abstract: In order to improve the file transfer speed between two smart mobile terminals, the point-to-point (P2P) file transfer system based on near field communication (NFC) is specifically designed and implemented. Using simple NFC data exchange format (NDFT) protocol, the message exchanging is implemented during two NFC devices. Both NFC and Bluetooth technology are employed in this system. The system front application (App) is developed by Android platform offered by Google. When the Android terminals is touched by each other, the communication links can be established, Bluetooth technology is used to implement file transfer through far distance. lots of experimental results show that this system is practicability and reliability, high efficiency and feasibility solution is provided for P2P file intelligence transfer system.

\section{Introduction}

Bluetooth and NFC technology is integrated in P2P file transfer system based on NFC. The system can achieve rapid file transmission between two intelligent mobile terminals. The communication $\mathrm{P} 2 \mathrm{P}$ program between the two NFC devices can be optimized[1].

P2P NFC communication is the application of Android Beam technology, that is to say, App is developed with Android system. The P2P communication mode has been defined in NFC forum, it can support the bi-directional transmission between two NFC devices and realize the P2P transmission of data [2]. The transmission files include pictures, video, audio, programs, and other Android Package (APK) [3]. In P2P communication mode, the two NFC devices participating in communication are called the initiating device (Initiator) and the target device (Target), respectively. The device between Initiator and Target can be exchanged, NFC P2P transmission rates include $106 \mathrm{kbit} / \mathrm{s}, 212 \mathrm{kbit} / \mathrm{s}, 424 \mathrm{kbit} / \mathrm{s}$. Due to limitation of the transmission speed, the P2P communication is suitable for the transmission of small files. Two NFC devices must be kept communication range less than $4 \mathrm{~cm}$ during transmission. Otherwise, the communication link is interrupted and the file transmission is failed [4]. In order to improve the transmission rate of the file and increase communication distance, the fast matching between two NFC devices is realized by the P2P file transfer system based on NFC, then the data transmission is realized with the Bluetooth technology [5].

\section{Overview}

P2P communication mode is one of the three working modes of NFC technology. In this mode, P2P data communication can be achieved between two NFC devices. P2P NFC communication is also known as the beam Android technology. This system uses beam Android to realize the establishment of the communication link between two mobile terminals, and uses the Bluetooth technology to realize the mutual communication between the files. Therefore, the file is transmitted more easily and fastly[6].

NFC technology is a technology of close range high frequency(HF) radio communication, which is based on radio frequency identification (Radio Frequency Identification, RFID) technology[7]. The communication distance must be kept less than 4 centimeters between devices and NFC tags or between two devices by NFC technology. Its working frequency is $13.56 \mathrm{MHz}$, and the working 
principle is electromagnetic induction coupling technology [8]. NFC electromagnetic induction coupling system and equivalent circuit diagram is shown in Figure 1.

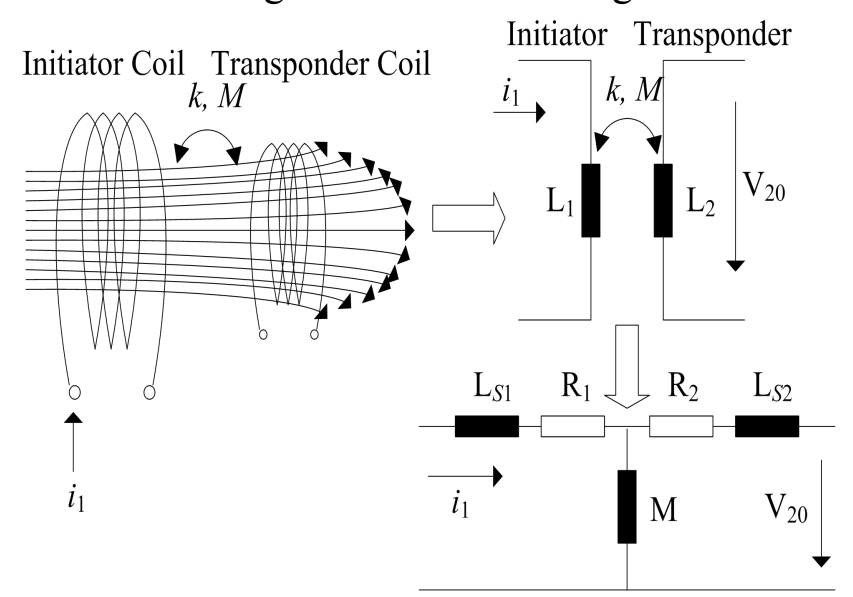

Figure 1 NFC electromagnetic induction coupling system and equivalent circuit diagram

In this NFC system, if the device relies on its own energy provided RF field to activate communication between origin and target, which is called active device. If the device relies on the RF field of other devices to provide energy and response, then it is called passive device. When data is interacting between the transmitter (initiator) and the responder (transponder), at least one side is the main device [9].

\section{Design}

\section{A. System architecture}

P2P file transfer system includes Initiator and Target. First, the role of Initiator and Target is selected for two NFC smart mobile terminals. When the two NFC phone is touched, the link is started to build, the selected file will pass through Bluetooth technology from Target to Initiator. When two NFC smart mobile terminals are interchanged, the file can be reversed from the new Initiator to the new Target. P2P file transfer system architecture diagram is shown in Figure 2.

B.P2p communication protocol

The top of the P2P NFC protocol stack is the logical link control (LLC) layer, which is used by logical link control protocol(LLCP). Simple NDEF exchange protocol (simple NDEF exchange protocol, SNEP) is above the LLC layer. Direct exchanging NDEF message is supported by NDEF between two NFC devices; NFC can support other protocol of high level and wide use by protocol binding. Connection handover protocol is used commonly by other protocols (CHP) [10]. P2P file transfer system is developed based on SNEP[11]. P2P NFC protocol stack is shown in Figure 3.

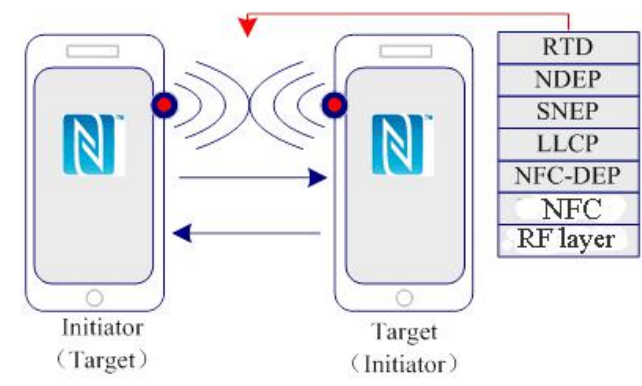

Figure 2 P2P file transfer system architecture diagram

\begin{tabular}{|c|c|c|}
\hline Ref Apps & \multicolumn{2}{|c|}{ Applications } \\
\hline Simple NDEF & NFC Forum & \\
Exchange & Registered Protocols & Other \\
\cline { 2 - 2 } Protocol & Protocols \\
\hline \multicolumn{2}{|c|}{ Logical Link Control Protocol } \\
\hline \multicolumn{2}{|c|}{ Digital Protocol } \\
\hline \multicolumn{2}{|c|}{ Analogue } \\
\hline
\end{tabular}

Figure 3 NFC P2P protocol stack

LLCP based on the IEEE 802.2 specifications, which defines the second layer protocol of the open system interconnect reference model (OSI)[12]. Limit data transmission of small application can be better supported by LLCP, such as the exchange of business cards or mutual recognition of magnetic stripe card number. SNEP is specially designed for P2P mode, which is intended to exchange 
information for NDEF messages during communication[13]. SNEP is a data transport protocol oriented link, its service port number is 0x04, the service name is "urn:nfc:snep". SNEP working mode is Request/Response. First of all, SNEP client sends SNEP request message to server for processing; then, SNEP server reply response to the SNEP client and inform the client processing results[14].

\section{C.P2P communication process}

Within the range of effective communication distance, when two NFC devices open the NFC function simultaneously, their LLC module will be the link activation state. In this stage, two NFC devices play two roles respectively: Initiator and Target. First, the Initiator sends a parameter exchange (PAX) packet to the Target, the packet is used to exchange configuration information for the LLC layer of two NFC devices[15]. The PAX packet is correspondingly processed after it is received by Target, if the processing is successfully completed; the LLC layer configuration information was transmitted to Initiator. When Initiator detects that the parameters of the LLC layer from Target is configured correctly, two NFC devices establish the logic link and start to work. When two NFC devices exceed the limited communication range, the deactivation link (DL) is triggered and the link is disconnected. The activation link work flow diagram is shown in Figure 4.

After the link is activated, link-oriented relation is established between two NFC devices, then exchanging data for each other. Client initiates the connection request to the server through the connection packets, when the connection packet is processed successfully by server, it will send complete packages to the client, the link is established successfully. Since then, the client and server can pass data through the information packets and the ready receive (RR) packets. Link oriented work flow diagram is shown in Figure 5.

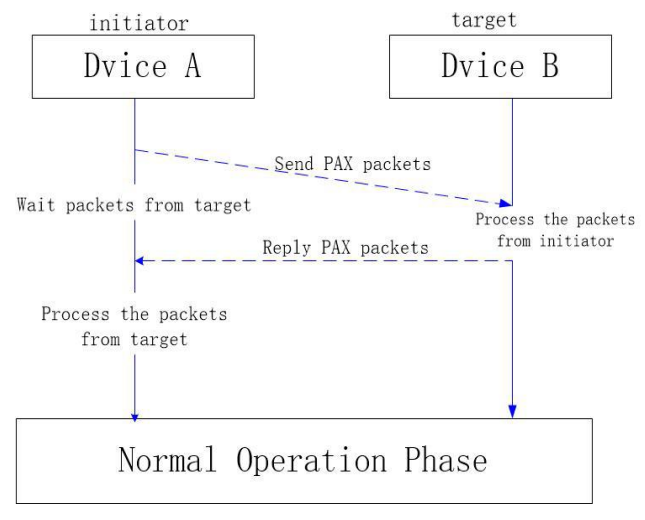

Figure 4 Link Activition workflow chart

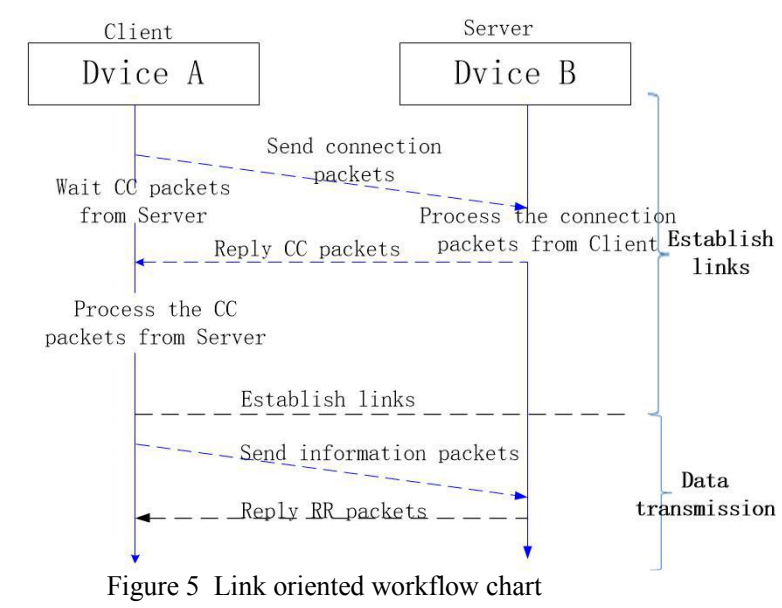

Figure 5 Link oriented workflow chart

When the communication link between two NFC devices is established, the system quickly connects with the Bluetooth service. As the Android platform provides a complete package for the Bluetooth communication protocol, the system directly calls the interface provided by Android to realize the service of the Bluetooth transmission.

\section{Implementation}

Application program interface(API) P2P NFC com-munication is known as beam Android, which allows for a simple data peer interaction between two Android devices. When the Initiator and the Target devices are within the effective range of the NFC communication, user interface(UI) is touched, then the information which selected by user will be transmitted from Target to Initiator.

By calling setNdefPushMessage() or setNdefPush-MessageCallback() method, the Android beam can be used App. The setNdefPushMessage() method sets the received NdefMessage object as a message to the beam. When these two devices are within the range of NFC communication, the message will be automatically sent. When the SetNdefPush-MessageCallback() method receive a callback containing createNdefMessage() method, and the Android smart mobile terminal is in the range of its launching data, the callback method will be called. 
An activity can only push a NDEF message. If the two methods are used at the same time, the setNdefPush-MessageCallback() method is more priority than setNdefPush-Message() method.

When the beam Android is started using, you first need to create a NdefMessage object that contains the NdefMessage, which is ready to be pushed to another device. Then calling setNdefPushMessage() method with NdefMessage type parameter or calling setNdefPushMessageCallback method in oncreate() method of activity, the purpose is to transfer the object that implements the interface NfcAdapter. Create-NdefMessageCallback.

In this paper, the beam Android technology is used to achieve the quick matching of two NFC devices, when the communication link is established, the system is connected with the Bluetooth. Since the Android platform provides a complete package for the Bluetooth communication protocol, the interface is directly called, and the class Bluetoothservice.java is defined to realize the service of Bluetooth transmission. After the consent receiving thread AcceptThread() is started, a connected single thread ConnectedThread () is established, the data is transferred through $\operatorname{read}()$ method, the transmission is canceled through cancel() method.

\section{Test result}

\section{A. Test environment}

In the P2P file transfer system testing, the communication is bidirectional, so NFC tablet and NFC phone are selected for P2P testing. P2P file transfer system test tool table is shown in Table 1.

Table 1 P2P file transfer system test tool

\begin{tabular}{|l|l|}
\hline \multicolumn{1}{|c|}{ Test tool } & \multicolumn{1}{c|}{ Illustration } \\
\hline NFC tablet PC & $\begin{array}{l}\text { Model, Google Nexus 7 } \\
\text { Version, Android 4.2.2 }\end{array}$ \\
\hline NFC smart phone & $\begin{array}{l}\text { Model, Google Nexus 6 } \\
\text { Version, Android 5.0 }\end{array}$ \\
\hline
\end{tabular}

\section{B.Test result and analysis}

The P2P file transmission App is installed dividedly in NFC mobile phones and NFC tablet. The phone is selected as Initiator, the tablet is selected as Target. If the NFC Tablet doesn't start, then two devices are keeping in a non-lock screen state. The App system main interface as shown in Figure 6 , four buttons on interface were used to get the machine video, audio, pictures and files list, click the button to select the transmitted file (for example, to enter the list of audio, select the first audio files to be transferred, the audio interface list is shown in Figure 7). The induction coil regions of NFC mobile phones and NFC tablet are approached, the NFC mobile phone screen is triggered by hand, the audio files in NFC mobile phone can be transmitted to the NFC tablet, P2P communication test process diagram is shown in Figure 8.

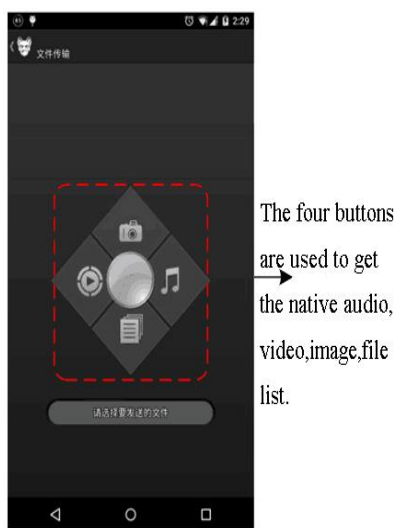

Figure 6 P2P communication APP test UI

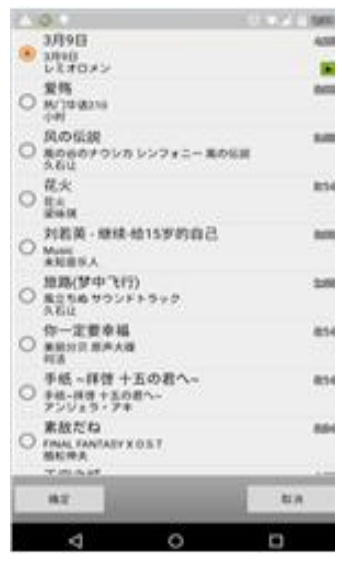

Figure 7 Audio list UI

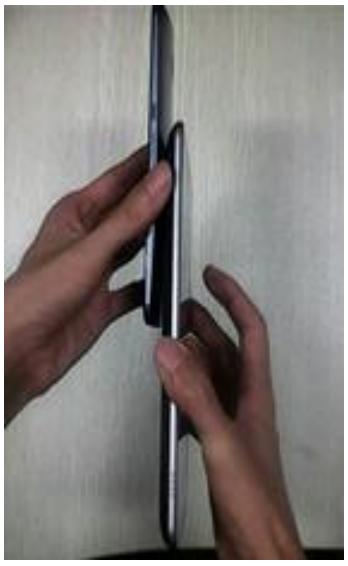

Figure 8 P2P communication test procedure chart 
In the process of file transmission, due to using Bluetooth technology, you can move the NFC mobile phone from the NFC tablet not to affect the file transmission process. The screen shot of $\mathrm{P} 2 \mathrm{P}$ communications file transmission process is shown in Figure 9, "the transmitting information" can be seen in the NFC mobile phone prompt column, the receiving beam can be seen in content NFC tablet prompt column. After the file is transmitted successfully, you can play the audio file on the NFC tablet.

The operation of the former paragraph is reversed, the files selected in the NFC tablet computer are transmitted to the NFC mobile phone successfully. This shows that two NFC devices have been tested through bi-direction $\mathrm{P} 2 \mathrm{P}$ communication. Using the P2P NFC file transfer system, the communication link can be realized only when the two NFC devices are touched, and the complex matching process of the Bluetooth is removed. Data is transmitted through Bluetooth technology, so the transmission rate is increased, and it successfully avoid the interruption of the communication process when the two NFC devices are removed.

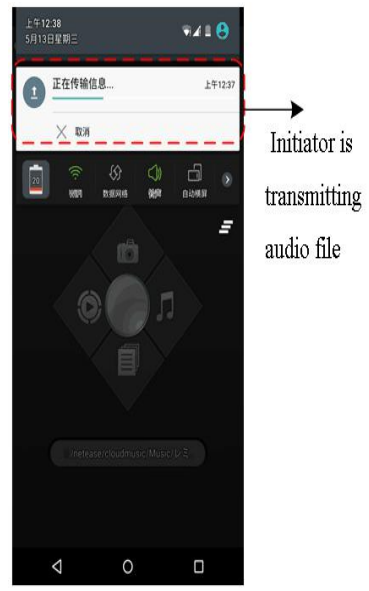

Figure 9(a) The screenshot of the Initiator is transmitting audio file

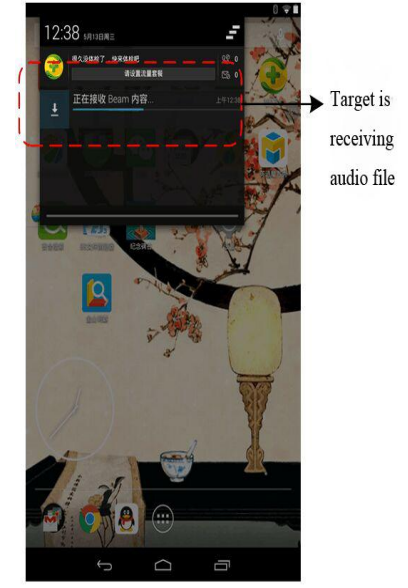

Figure 9(b) The screenshot of the Target is receiving audio file

\section{Conclusion}

The P2P file transmission system adopts NFC technology to realize the matching connection of the two devices, and the data transmission is realized through the Bluetooth technology. The system makes full use of the communication and transmission advantages of NFC and Bluetooth, the method of organically merge of the NFC and Bluetooth technology is successfully used to achieve P2P file transmission of further distance, which provides an efficient and convenient solution for intelligent mobile terminal file transmission.

\section{References}

[1] E.Harjula, Energy-efficient Peer-to-Peer Networking for Constrained-Capacity Mobile Environments, 2016.

[2] B.Schlomann, W. Eichhammer, L. Stobbe, "Energy saving potential of information and communication technology", International Journal of Decision Support Systems, vol. 1, no. 2, pp. 152-163, 2015.

[3] "Internet Trends 2015-Code Conference", KPCB, 2015, [online] Available: http://www.kpcb.com/internet-trends.

[4] "Wikipedia", Web Server, 2014, [online] Available: http://en.wikipedia.org/wiki/Web_server.

[5] M. Amad, D. Aïssani, A. Meddahi, M. Benkerrou and F. Amghar, De Bruijn Graph based solution for lookup acceleration and optimization in P2P networks, Wireless Personal Communications 85: (3) ((2015) ), 1471-1486. doi:10.1007/s11277-015-2851-y. 
[6] Y. Benkler, "Degrees of Freedom Dimensions of Power", Daedalus Winter 2016, vol. 145, pp. $18-32,2015$.

[7] J. Bonneau, A. Miller, J. Clark, A. Narayanan, J. A. Kroll, E. W. Felten, "SoK: Research Perspectives and Challenges for Bitcoin and Cryptocurrencies", IEEE Symposium on Security and Privacy. IEEE Computer Society, pp. 104-121, 2015.

[8] J. Benet, IPFS - Content Addressed Versioned P2P File System, vol. abs/1407.3561, 2014

[9] "Measuring the Information Society Report", International Telecommunication Union (ITU) Report, 2015.

[10]ISO/IEC18092, Information technology - Telecommunications and information exchange between systems - Near Field Communication Interface and Protocol (NFCIP-1)[S].

[11] Serfass D, Yoshigoe K. Wireless Sensor Networks using android virtual devices and Near Field Communication peer-to-peer emulation[C]// Southeastcon, 2012 Proceedings of IEEE. IEEE, 2012,pp.1 - 6 .

[12]Urien P. LLCPS: A new security framework based on TLS for NFC P2P applications in the Internet of Things[C]// Consumer Communications and Networking Conference (CCNC), 2013 IEEE. IEEE, 2013,pp.845- 846.

[13] Castiglione A, De Prisco R, De Santis A, Fiore U, Palmieri F (2014) A botnet-based command and control approach relying on swarm intelligence. J Netw Comput Appl 38:22-33.

[14]He J, Yang Y, Wang X, Zeng Y, Tang C (2014) Peersorter: classifying generic p2p traffic in real-time. In: 2014 IEEE 17th International conference on computational science and engineering (CSE),IEEE, pp 605-613.

[15]Kim JY, Chung KY, Jung JJ (2014) Single tag sharing scheme formultiple-object RFID applications. Multimedia Tools Appl 68(2):465-477. 